\title{
Automated Services for Agro-farms using Internet of Things (loT)
}

\author{
Pamidi Srininvasulu, PhD \\ DVR \& Dr. HS MIC College of \\ Technology, Kanchikacherla, \\ A.P, India, 521180
}

\author{
M. Sarath Babu, PhD \\ DVR \& Dr. HS MIC College of \\ Technology, Kanchikacherla, \\ A.P, India, 521180
}

\author{
R. Venkat \\ DVR \& Dr. HS MIC College of \\ Technology, Kanchikacherla, \\ A.P, India, 521180
}

\begin{abstract}
The Food and Agricultural Organization of the UN (FAO) predicts that the global population will reach 8 billion and 9.6 billion by 2025 and 2050 respectively. In order to meet the increasing demand for food, production must inevitably increase by 70 percent by 2050 . According to a recent report by the UN's Intergovernmental panel on Climate change (IPCC), there will be a number changes of climate that are likely to affect the agriculture and food sectors. In the present work, an attempt has been made to propose a framework for farming which involves a wide range of technologies that include natural farming methods, online services on current marketing, GPS Services, Wireless Sensor Networks(WSN), Internet of Things (IoT) and big data, there by the technologies will yield optimum profits. Information and Communication Technologies (ICT)-based decision support system, backed up by real-time data, can additionally provide information concerning all aspects of farming at a level of granularity that was not previously achieved. At the end of the day, this framework enables to go for better decisions, by reducing the use of resources and yield of waste through maximum efficiency and thereby maximum profits attained in agro-based operations.
\end{abstract}

\section{General Terms}

Internet of Things, Wireless Sensors, Controllers, Automation, Services, Farming, Pest Control, Organic farming, Environment monitoring, Data acquisition, SMS Messages, Mobile communication, GPS Services, Big data, ICT Technologies, Soil health protection, Agro-products, Marketing.

\section{Keywords}

GPS, Web Services, Internet of Things, Wireless Sensor Network, ICT Tools, Big Data, Agro Farm

\section{INTRODUCTION}

The history of agriculture has played a major role in human history, as agricultural progress has been a crucial factor in worldwide socio-economic change. Division of labor in agricultural societies made commonplace specializations rarely seen in hunter-gatherer cultures, which allowed the growth of towns and cities, and the complex societies we call civilizations. When farmers became capable of producing food beyond the needs of their own families, others in their society were free to devote themselves to projects other than food acquisition.

Over 200 years ago, 90 percent of the population lived on farms and produced their own food to eat. But today, only five percent of the population produces the food, including fruits, vegetables, meats and dairy, that everyone eats (Prax, 2010). That's a large change in the amount of people associated with producing food and making sure that everyone has enough to eat.

Farmers use technology to make advances in producing more food for a growing world. Through the use of technology, each farmer is able to feed 155 people today, compared to 1940, when one farmer could feed only 19 people (Prax, 2010). Farmers use technologies such as motorized equipment, modified housing for animals and biotechnology, which allow for improvement in agriculture [8]. Better technology has allowed farmers to feed more people and requires fewer people to work on farms to feed their families.

Changes in equipment have made a large impact on the way farmers are able to farm and grow food. In the past, farmers would have to do field work by hand or with bull-drawn equipment. This work would take a long time to complete, which meant that farms were smaller because farmers could only work so much land. Bulls were not very fast, and they would tire out so farmers would have to let the bulls rest. Today, most farmers use tractors and other motorized equipment to help with field work. Tractors are larger and move faster than bulls, so farmers are able to work on more land and grow more food. Tractors still need to be taken care of because they can break down just like a car. But they can run for longer periods of time and work when the farmer needs them.

The Food and Agricultural Organization of the UN (FAO) predicts that the global population will reach 8 billion people by 2025 and 9.6 billion people by 2050 . In order to keep pace, food production must increase by 70 percent by 2050.According to a recent report by the UN's Intergovernmental panel on Climate change (IPCC), there will be a number of effects of climate change on agriculture [4].

Given the high pressure on agricultural resources, IoT can serve as a way to help farmers make informed decisions and maximize yields from their resources. IoT applications for agriculture enable farmers to, amongst other activities, remotely measure soil conditions, monitor equipment, track the weather, and assess the health of livestock and crops, all via mobile phones, computers and other devices. IOT can be used to send and receive data about temperature, weight, location and any number of other agricultural factors, as well as requests to each other and to central management systems, autonomously. The stakeholders in the IOT value chain include module vendors, connectivity providers, IOT platform and application providers, device platform providers, mobile operators, aggregators and mobile virtual network operators (MVNOs). The information is collected through IOT modules mounted on the assets and transmitted via connectivity providers (mobile operators in this case). This information is then received by system integrators and solution providers 
which gather and process the data, to be finally displayed via mobile or web applications to the end users [3].

Smart agriculture aims to optimize the yield per unit of farming land by using the most modern means in a continuously sustainable way, to achieve best in terms of quality, quantity and financial returns.

Smart agriculture makes use of a range of technologies that include GPS Services, sensors and big data to optimize crop yields. Rather than replace farmer expertise and gut feeling, ICT-based decision support system, backed up by real-time data, can additionally provide information concerning all aspects of farming at a level of granularity not previously possible. This enables better decisions to be made, resulting in less waste and maximum efficiency in operations.

Internet of Things is based on sensor technologies [5] whose use is well established in other industries, e.g. Telematics for fleet management, environmental monitoring for pollutants, eHealth monitoring in patients, and building management for farm and so on. For all IoT implementations, IT Systems gather, collate, analyze the data and present it in such a way as to initiate an appropriate response to the information received. For farmers and growers, a wide variety of information regarding soil and crop behavior, animal behavior, machine status, storage tank status emanating from remote sites is presented for action by the farmer.

The agriculture sector has been playing a major role in history, since it has been a crucial factor in the global socio-economic revolution. The division of labor in agricultural societies was very common whereas the same was rarely observed in hunter-gatherer cultures that allowed the growth of towns and cities, into complex societies and the so called civilization that we are in. When farmers were capable of producing food beyond the needs of their families, the others in the society were free to devote themselves to projects other than food acquisition.

\section{IMPORTANCE OF THE AGRO FARMS}

The Food and Agricultural Organization of the UN (FAO) predicts that the global population will reach 8 billion people by 2025 and 9.6 billion people by 2050. In order to keep pace, food production must increase by 70 percent by 2050 . Briefly

- Population size and commensurate food demand are increasing;

- Competition for food, land, water, energy, as well as carbon storage, is intensifying; and

- Degradation of natural resources is expanding;

This has led to the recognition that these challenges must be addressed together, rather than in isolation from each other. Yet, policies have often remained unaligned and institutional/financing arrangements fragmented.

Modern agriculture is no longer limited to development of cultivation tools but focuses on automation and control, advanced local and remote sensing, precision, post harvest treatment of biological materials, machine-soil interaction, energy efficient development, and much more. The proposed agricultural engineering work deals with various topics related to mechanics and to sensing in both to classic agricultural engineering, as well as to all fields of environmental engineering and water systems [11].

\section{METHODOLOGY}

The digital divide between rural and urban India is slowly but surely decreasing with farmers getting familiar with mobile and internet applications. But, to the best of our knowledge, there is no application, which is personalized, catering to the specific needs of a farmer. The proposed solution attempts to do just that. A wide range of services are provided to the farmer and the gram panchayat users of a locality who are closely associated with the farmers. All these services are personalized, meaning, the information appearing on the user interface, are relevant to the farmer who uses the application. Personalization of the farmer services is achieved by capturing the location of the farm, so that data relevant to that location can be given, rather than general information $[6,7]$. The various personalized services provided in the proposed solution are described in the subsequent sub-sections. The following modules are proposed to develop:
a. Registration Service
b. Yield Prediction Service
c. Market and Storage Locator Service
d. Expert Advice Service
e. Crop Monitoring Service

\subsection{Registration Service}

This service enables new farmers to register into the system. The details captured are - farmer's land location details, soil type, crops grown, amount invested by the farmer for seeds, pesticides and fertilizers in last three years, the crop yield, income or loss incurred for each crop etc. This 'history' data gathered is important for the other farmer friendly services of the system.

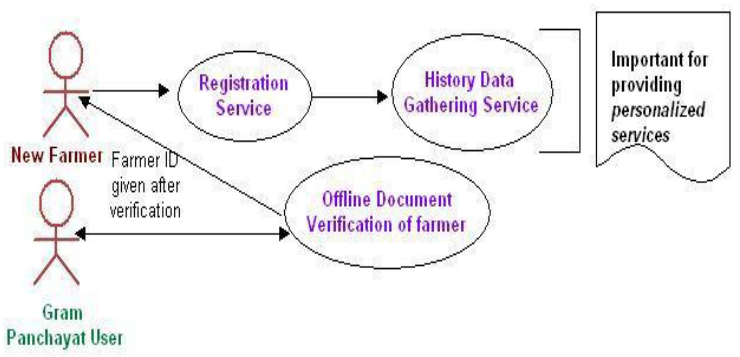

Figure 1. Use Case depicting the Registration Service

\subsection{Yield Prediction Service}

This predicts the yield a farmer can expect. A 'Regression Model', is used where data is utilized to identify relationships among variables. These relationships are used for predictions. Here, a multiple regression model [2], with many independent variables is used. The following predictions are done, based on two profiles of the farmer's land, namely, soil profile (soil type, nutrients present) and land profile (land area and location)

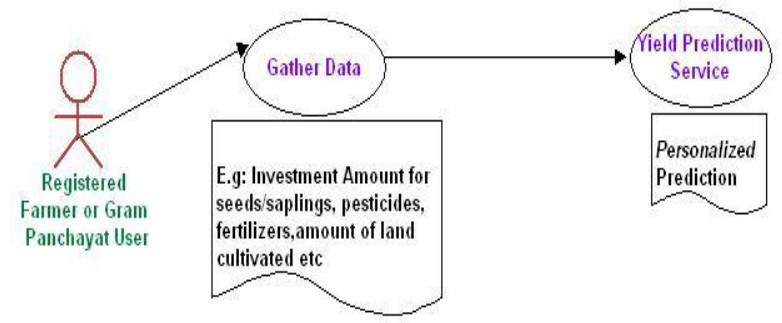

Figure 2. Use Case depicting the Yield Prediction Service 
i. The investment on fertilizers, seeds etc for a given soil and land profile, to get 'p\% 'yield,' $\mathrm{n}$ months later, for a specific crop.

ii. The yield that can be got, for a farmer's soil and land profile with the amount of fertilizers and pesticides applied.

iii. A comparative analysis of the yield that can be got for a particular soil and land profile, if different types of crops fit for that profile are cultivated, instead of the regular type.

\subsection{Market and Storage Locator Service}

This service is used to locate the local and export markets, near the farmland, for the farmer to sell and store his produce hygienically.

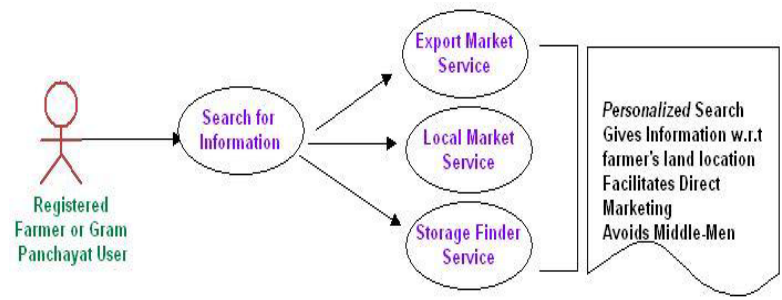

Figure 3. Use Case depicting the Market and Storage Locator

This service is personalized for each farmer, based on the location of his farmland, and presents relevant results useful to him. This is one step towards direct marketing of the agroproducts, avoiding the current corrupt chain of middle-men [1, 2]. They are like vultures, demanding high brokerage fees for facilitating selling the agro-products, cultivated by the farmer with his sweat and blood. Sometimes, this brokerage fees is so high that the farmer has no option but to hoard his produce in his backyard, in unhygienic conditions, prone to insects and pests.

\subsection{Expert Advice Service}

This service aims to bridge the gap between the vast amount of knowledge with agricultural scientists and the percolation of this know-how to the farmers. Currently, in India, there is vast amount of research done by agriculturalists, focused on addressing the problems of the farmers, but the outcomes and best practices of this research does not reach the end beneficiary. As shown in Figure 4

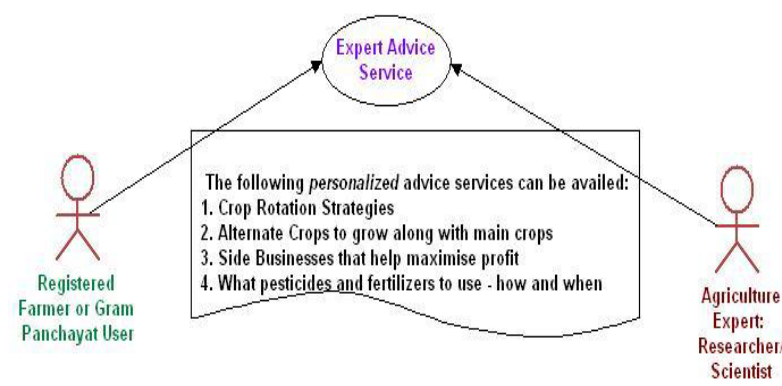

Figure 4. Use Case depicting the Expert Advice Service

\subsection{Crop Monitoring Service}

In this service, the agricultural expert gets to view the various stages of crop cultivation, starting from preparation of the cultivable land, through pictures uploaded from the mobile application provided by the system [3]. This application is available with the end user, namely, farmer, gram panchayat worker or cooperative society personnel, all of whom are stake-holders for the farmland and its produce. As shown in Figure 5, the experts, now facilitated with the actual pictures of the crop are in a better position to impart their expert advises.

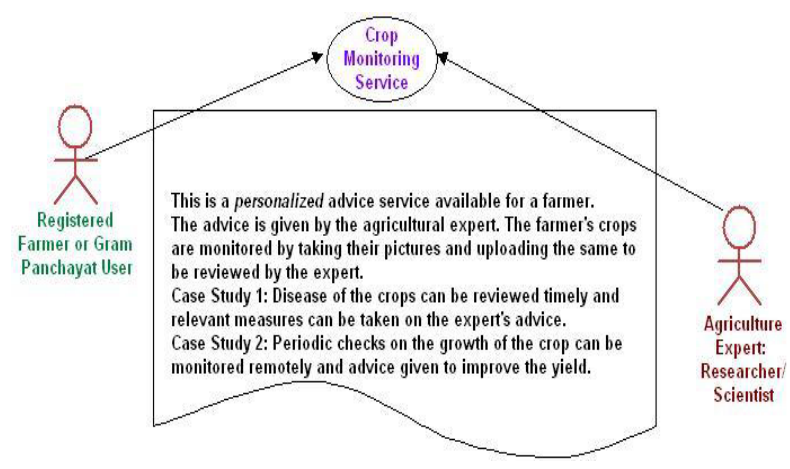

Figure 5. Use Case depicting the Crop Monitoring Service

The following items are managed by the digital farming system.

$>$ Data, information, and knowledge

$>$ Software needed to manage holdings

> Interfaces to algorithms and physical resources to support acquisition of data and their transformation into information and knowledge, storing the protocols to interact with other facilities

\section{CONCLUSION}

This paper objective is to improve the yielding and indirectly to improve the greenery. It is possible to improve the soil health conditions by following natural farming methods and avoiding using chemicals and feticides $[9,10]$. The health of the soil in which our food is grown is intimately connected to our health, not to mention the environment as a whole. Our water quality wasn't getting better, and the farmers were going broke.

The worst thing about it is we couldn't help them. Simply stated, soil health is the soil's ability to function, to sustain animals, plants, humans, and our climate. The healthier the soil is, the more it functions properly. Keeping the soil covered, having living root, bringing diversity, and integrating animals - those are principles to make soil healthy and functioning."

\section{REFERENCES}

[1] Robert Jensen, "Information, Efficiency and Welfare in Agricultural Markets", in the proceedings of the 27thInternational Association of Agricultural Economists Conference, Beijing, China, Aug 16 - 22, 2009, pp 1 29.

[2] Agmarknet: A Step towards globalization of Indian agriculture, Web Page retrieved on 12th Feb 2012, 06.12 pm from http://agmarknet.nic.in/.

[3] Jadhav and Shinde, "Web Based Information System for Agriculture", In International Journal of Innovative Technology and creative engineering, Vol 1, No.2, Feb 2011

[4] http://www.fao.org/myanmar/news/detailevents/en/c/385218/ 
International Journal of Computer Applications (0975 - 8887)

Volume 154 - No.11, November 2016

[5] https://www.beechamresearch.com/files/BRL\%20Smart $\% 20$ Farming\%20Executive\%20Summary.pdf

[6] http://www.sensegrow.com/blog/iot-remote-monitoringsmart-farms

[7] www.ijstr.org/files/nov2012/1350668330IBM_SMART \%20FARMING.doc
[8] http://cee.technion.ac.il/eng/Templates/showpage.asp?D $\mathrm{BID}=1 \& \mathrm{LNGID}=1 \& \mathrm{TMID}=84 \& \mathrm{FID}=539$

[9] http://mesell.biz/myblog/?p=2699

[10] https://www.organicconsumers.org/news/scientistreveals-simple-tips-improving-soil-health

[11] https://en.wikipedia.org/wiki/Water_pollution 